\title{
A comparison of techniques to address the frequency of Helicobacter pylori positive dyspeptic patient
}

\author{
Priyatam Khadka ${ }^{1 *+}\left(0\right.$, Ganesh Chapagain ${ }^{1+}$, Govinda Maharjan ${ }^{1}$ and Prakash Paudyal ${ }^{2}$
}

\begin{abstract}
Objectives: The study was aimed to compare the diagnostic techniques, for the detection of Helicobacter pylori infection, available in low-income countries, where molecular testing is not available or inaccessible to anyone.

Results: Of total enrolled patient, with the mean age of $41.4 \pm 13.33$ years, 24 ( 14 female; 10 male) were diagnosed to have been infected. The diagnosis was established based upon the gold standard test [either two of three tests: Rapid Urease Test (RUT), culture and histological examinations]. Of clinical presentation, the epigastric pain (75\%) was the commonest; nevertheless, the endoscopic findings had shown an equivocal specificity since the larger percentile (58.3\%) reported as normal findings, in a presumed dyspepsia. Based on the premise-with calculated sensitivity, specificity, and predictive values; the accuracy order observed as histology > RUT > serology > stool antigen test, in $H$. pylori detection from the clinical samples. The accuracy order of the diagnostic test may vary depending upon the laboratory settings and study population. Therefore, in reference to low-income countries, the clinicians must resort any available positive test so that the supporting positive rudiments would be an ancillary in augmenting the diagnostic accuracy.
\end{abstract}

Keywords: Diagnostic test, Functional dyspepsia, Helicobacter pylori, Low-income countries

\section{Introduction}

Helicobacter pylori, a definite biological carcinogen as defined by WHO 1994, is of major concern today; because of its causal gastrointestinal diseases including gastritis, duodenal and gastric ulcers, non-ulcer dyspepsia, gastric adenocarcinoma, and lymphoma $[1,2]$. H. pylori is one of the most successful human pathogen, since many chronic viruses infect close to $100 \%$ of world population [3]. Meanwhile, relating the clinical presentations, nearly $5 \%$ of functional dyspepsia may be attributable to the pathogen [4].

Dyspepsia is an extremely common disorder in an otherwise healthy individual comprising an assorted range of

\footnotetext{
*Correspondence: khadka.priyatam@gmail.com

${ }^{\dagger}$ Priyatam Khadka and Ganesh Chapagain contributed equally to this work

${ }^{1}$ Trichandra Multiple Campus, Tribhuvan University, Ghantaghar, Kathmandu, Nepal

Full list of author information is available at the end of the article
}

chronic upper abdominal symptoms which resembles the different gastrointestinal disorders: reflux disease, irritable bowel disease, gallbladder, and pancreatic dysfunction, and celiac disease [5, 6].

The chronic or recurring epigastric pain or discomfort centered in the upper abdomen for which no associated organic disease can be determined, classically described as the functional dyspepsia [6]. The clinical diagnosis, in general, presumed as functional dyspepsia or investigated dyspepsia when other possible gastrointestinal diseases along with a macroscopic lesion observed on upper endoscopy were excluded [6]. The conditions appear more frequently in the clinical practices i.e. of the global prevalence $7-45 \%$ in an uninvestigated functional dyspepsia [7]. Hence, an early and precise diagnosis of the etiology, in a presumed dyspeptic patient is crucial for therapeutic interventions. At this time, a myriad of invasive and non-invasive tests are implicated in diagnosis; however, applicability testing, clinical circumstances and cost-effectiveness of the test strategy are 
obligatory-particularly in low-income countries where molecular testing is not available or unreached to anyone.

\section{Main text \\ Materials and methods \\ Patient selection}

Between, January and June 2018, the consecutive outpatients receiving diagnostic endoscopy at the Department of Gastroenterology, Nepal National Hospital for unexplained dyspepsia were enrolled in our study.

\section{Inclusion criteria}

The patients, equal number of male and female, visiting Nepal National Hospital with uninvestigated symptoms of dyspepsia with gastrointestinal symptoms: upper abdominal pain or discomfort, bloating, nausea and vomiting, or early satiety, were included in the study.

\section{Exclusion criteria}

The patient who underwent partial or complete gastrectomy or major abdominal surgery, those with a history of peptic ulcer organic and metabolic diseases, use of steroids or non-steroidal anti-inflammatory drugs, or those with $H$. pylori eradication therapy or under any antimicrobial therapy within past 4 weeks were excluded from the study.

The research protocol was approved by the local ethical committee of the Nepal National Hospital. A written informed consent was obtained from every patient prior endoscopy, and sample collection.

\section{Endoscopy and biopsy sampling}

After an overnight fast, the endoscopic procedures were performed by the Gastroenterologist. Four gastric biopsies (two from the antrum and two from the corpus) were taken from each patient. Of total four samples, two of them-one from corpus from antrum-were fixed in $10 \%$ formalin in different vials and were sent for histopathological examination. Similarly, two of them-one from corpus from antrum were sent to the microbiology laboratory of the Nepal National Hospital for culture and urease test following aseptic measures.

\section{Microbiological culture and Rapid Urease Test (RUT)}

The RUT was performed from the non-commercial validated test. The reagent preparation was done as per standard microbiological methods recommended by American Society of Microbiology (ASM). In brief: $1 \mathrm{~mL}$ distilled water +1 drop of $1 \%$ phenol red $+100 \mathrm{mg}$ urea (Hi media, India), prepared just before endoscopy. The biopsy samples were stabbed, of two gastric biopsy specimens, the biopsy from antrum was placed in $1 \mathrm{~mL}$ urease broth for RUT; while biopsy from corpus was placed in Stuart's transport medium for a microbiological culture.

Within a few minutes, the samples were processed, with grinding and streaking aseptically, with recommended methods. In brief, for microbial culture, the specimen was inoculated in Brain Heart Infusion (BHI) agar supplemented with: sheep blood (10\%), amphotericin $(5 \mathrm{mg} / \mathrm{L})$, cefsulodin $(5 \mathrm{mg} / \mathrm{L})$, trimethoprim lactate $(5 \mathrm{mg} / \mathrm{L})$, and vancomycin $(10 \mathrm{mg} / \mathrm{L})$; incubated under micro-aerophilic condition $\left(5 \% \mathrm{O}_{2}, 10 \% \mathrm{CO}_{2}\right.$, and $85 \% \mathrm{~N}_{2}$ ) at $37{ }^{\circ} \mathrm{C}$ for 7 days. As for the presumptive bacterial growth monitoring, the minute colonies were stained on gram stain and observed for the gram-negative curved bacilli. Further, identification of growth of the bacteria was done following standard microbiological methods recommended by American Society of Microbiology [8, 9].

For RUT, the specimen was inoculated in $1 \mathrm{~mL}$ prepared urease broth for $2 \mathrm{~h}$ at room temperature and observed for pink color on the positive test.

\section{Histological examination}

For histological examination, the specimens were fixed with $10 \%$ formalin (not more than a week), embedded in paraffin, stained with Giemsa then observed microscopically for spiral bacteria.

\section{Serology}

On the same day as biopsies from patients undergoing endoscopy, $1 \mathrm{~mL}$ of sera was collected, aliquoted, and stored at $-20{ }^{\circ} \mathrm{C}$ until used. The serological assay for detection of IgG antibodies against $H$. pylori was performed by a commercial $H$. pylori IgG ELISA kit (IBL, Hamburg, Germany) according to the manufacturer's instructions. The cutoff value was assessed as per the manufacturer's manual for the positive or negative test. As anti-H. pylori immunoglobulin (Ig) G titers were $>12$ $\mathrm{U} / \mathrm{mL}$, the results were interpreted as positive, negative if they were $<8 \mathrm{U} / \mathrm{mL}$, and equivocal if they were between 8 and $12 \mathrm{U} / \mathrm{mL}$.

\section{Helicobacter pylori stool antigen test ( $\mathrm{HpSAg}$ )}

The stool antigen test for $H$. pylori was performed, as per the manufacturer instructions, in ImmunoCard STAT! HpSAg-test kit (later ImmunoCard) Meridian Bioscience Europe-based on monoclonal H. pylori antibodies and a lateral flow chromatography technique. In brief, a small portion (approx. 5-6 $\mathrm{mm}$ diameter) of stool specimen was transferred in sample diluents vial and mixed thoroughly. The diluted stool sample was dispensed to the sample well of the test cassette; was observed for pinkred line in the reading window on positive test after incubation of $5 \mathrm{~min}$ at room temperature. 
The sensitivities and specificities of the tests were calculated assuming the results (from culture, RUT, histopathological examinations) as gold standard in diagnosis of $H$. pylori infection [10]. If 2 of 3 tests were positive, the patients were considered to be infected with $H$. pylori (true positive); as if all three tests found negative were considered of not having $H$. pylori infection (true negative).

\section{Results}

\section{Patient demographics}

During the study period, out of 90 patients-with an uninvestigated symptom of dyspepsia and/or having gastrointestinal symptoms-enrolled as study subjects. As urea breathe test was not performed in our study (culture, RUT, histopathological examinations) were considered as gold standard for determination of sensitivities and specificities of the tests. Relying upon the stated criteria-at least two of three tests are positive; 24 (26.6\%) of the patients were diagnosed as the positive cases. Of reported positive cases, $14(58.33 \%)$ were female and 10 $(41.7 \%)$ male. The age ranged from 20 to 80 years, with mean age $41.4 \pm 13.33$ years.

\section{Clinical presentation and endoscopic findings}

Relating clinical presentations to the infection acquisition; the commonest, being the epigastric pain (75\%). However, dyspeptic symptoms (45.80\%), nausea and vomiting (29\%), alteration of bowel habits (16.6\%), and loss of appetite $(12.5 \%)$, were found as a non-specific clinical presentation in $H$. pylori-positive patients, as shown in Table 1(i).

The endoscopic findings had shown an equivocal specificity; since the larger percentile was reported as normal findings $(58.3 \%)$. The patients with ulcers (50\%), duodenitis (26.3\%), malignancies (25\%), and gastritis (16\%); however, found positive for the infection, as shown in Table 1(ii).

\section{Comparative diagnostic methods}

The biopsy based and serological assay based diagnostic test were analyzed for their sensitivities, specificities, predictive values, and accuracy in relation to the presumed gold standard (culture, RUT, histopathological examinations i.e. at least two of three test). The comparisons of different methods in diagnosis of $H$. pylori infection are shown in Table 2.

\section{Discussion}

Approximately, a half of the world population found traumatized with the $H$. pylori infection; regrettably, $90 \%$ of the estimated prevalence in developing countries [11-13]. It has been observed, in reference with a myriad of epidemiological studies, the significant differences in prevalence across the world; which relies particularly on socioeconomic conditions like overcrowding, poor sanitation, hygiene and behavior traits of the patient $[11,14-16]$. In an aforementioned epidemiological study, the prevalence of $H$. pylori in dyspeptic patients reported $38.4 \%$, based on the PCR findings, in Nepal [17]. However, the accessibility of molecular

Table 1 The clinical presentation and endoscopic findings in the presumed dyspeptic patient

\begin{tabular}{|c|c|c|}
\hline Clinical presentation & No. of case (\%) & $\begin{array}{l}\text { H. pylori } \\
\text { positive case } \\
(\%)\end{array}$ \\
\hline \multicolumn{3}{|c|}{ (i): Clinical presentation in presumed H. pylori positive dyspeptic patient } \\
\hline Alternation of bowel habit & $24(26.6)$ & $4(16.6)$ \\
\hline Abdominal pain & $81(90)$ & $18(75)$ \\
\hline Dyspeptic symptoms & $66(73.3)$ & $11(45.8)$ \\
\hline Nausea and vomiting & 69 (76.6) & $7(29)$ \\
\hline Loss of appetite & $41(45.5)$ & $3(12.5)$ \\
\hline Endoscopic findings & No. of case (\%) & $\begin{array}{l}\text { H. pylori } \\
\text { positive (\%) }\end{array}$ \\
\hline \multicolumn{3}{|c|}{ (ii): Endoscopic findings in H. pylori positive case } \\
\hline Normal & $12(13.3)$ & $7(58.33)$ \\
\hline Gastritis & $49(54.4)$ & $8(16.32)$ \\
\hline Duodenitis & $19(21.1)$ & $5(26.3)$ \\
\hline Ulcers & $6(6.6)$ & $3(50)$ \\
\hline Malignancy & $4(4.4)$ & $1(25)$ \\
\hline
\end{tabular}

Total enrolled case $=90$; total $H$. pylori positive case $=24$ 
Table 2 Comparison of different methods in the diagnosis of $H$. pylori infection

\begin{tabular}{|c|c|c|c|c|c|c|c|}
\hline \multirow[t]{2}{*}{ Test } & \multicolumn{2}{|c|}{ Gold standard } & \multirow[t]{2}{*}{ Sensitivity } & \multirow[t]{2}{*}{ Specificity } & \multirow{2}{*}{$\begin{array}{l}\text { Positive } \\
\text { predictive value } \\
(\%)\end{array}$} & \multirow{2}{*}{$\begin{array}{l}\text { Negative } \\
\text { predictive value } \\
(\%)\end{array}$} & \multirow[t]{2}{*}{ Accuracy (\%) } \\
\hline & Positive & Negative & & & & & \\
\hline Histology & 21 & 69 & 80.9 & 94.2 & 80.90 & 94 & 91.10 \\
\hline Rapid Urease Test (RUT) & 34 & 56 & 82.3 & 87.5 & 80.00 & 86 & 85.50 \\
\hline Serology (IgG ELISA) & 66 & 24 & 89.3 & 54.1 & 84.20 & 65.00 & 80 \\
\hline Stool antigen test (HpSAg) & 39 & 51 & 53.8 & 88.2 & 78 & 71 & 73.30 \\
\hline
\end{tabular}

testing, in most of the laboratory settings-principally in low-income countries, is beyond the reach of anyone. Hence an elucidation of all possible direct and indirect tests, with their calculated sensitivities, specificities, predictive values, and accuracies is necessary while opting as the diagnostic test.

An equal number of the male and female, although, enrolled in the study; larger number of the female having H. pylori infection was observed. The finding was consistent with the study of Miftahussurur et al. who found a similar female preponderance in dyspeptic Nepalese population [17].

Referring to our findings, on equating clinical presentation to the positive case: $75 \%$ had epigastric pain, $45.8 \%$ had dyspeptic symptoms, alternation of bowel (16.6\%). El-Omer et al., however, in their study has shown: 80-90\% of the dyspeptic patients have associated symptoms of epigastric pain, anorexia, nausea, vomiting, early satiety and regurgitation [18]. In contrast, Srinavas et al. reported the associated symptoms: epigastric pain (28\%), nausea $(0.1 \%)$, vomiting $(23.2 \%)$, early satiety $(0.18 \%)$, in the presumed $H$. pylori-infected patient [19].

Of total case enrolled, 12 of them had normal endoscopic findings; however, 7 (58.3\%) found positive of harboring the infection. The percentile/findings were in line with the study by Okello et al. where he reported $51 \%$ patient with negative endoscopic findings in $H$. pylori positive cases [20]. Besides, among patients with positive endoscopic findings, inflammatory lesions like ulcers (50\%) and duodenitis (26.3\%) observed more frequently.

Among the diagnostic test implicated in our study, the histological examination reveals the higher accuracy $(91.10 \%)$ with notable sensitivity $(80.9 \%)$ and specificity (94.2\%); the stool antigen test, nevertheless, shows lower accuracy (73.3\%) with limited sensitivity (53.8\%) and specificity (88.2\%). In an epidemiological study, Miftahussurur et al. reported as the overall accuracy (97.3\%), sensitivity (92.7\%), specificity (100\%) for histology; the findings are accordance with that our premises [17]. Similarly, Khalifehgholi et al. revealed, the accuracy, sensitivity, specificity for the histological test was $86.8 \%, 95.6 \%, 77.8 \%$ respectively. However, the stool antigen test compared to ours, Khalifehgholi et al. reported of higher accuracy (80.2\%), sensitivity (73.9\%), specificity $(86.7 \%)$, in positive $H$. pylori cases [10].

Similarly, RUT reveals the accuracy (85.5\%) with noteworthy sensitivity (82.3\%) and specificity (87.52\%); IgG serology (ELISA), nonetheless, shows the $(80 \%)$ accuracy with limited sensitivity $(89.3 \%)$ and specificity (54.1\%). Nevertheless, in a similar type of study, Khalifehgholi et al. reported the higher accuracy, sensitivity, specificity: $97.8 \%, 95.6 \%, 100 \%$ respectively for RUT; nevertheless, for IgG serology the (73.6\%) accuracy, sensitivity (91.3\%) and specificity (55.6\%) was elucidated [10].

Based upon the premise, the accuracy order of the diagnostic methods observed as histology $>$ RUT $>$ serology $>$ stool antigen test. The order, however, had found variable in different cohort studies; possibly due to the pathogen variant and immune response to the infection of different study population [10].

Currently, urea breath test with improved infrared spectrometers have shown an extra advantage of low cost with augmented sensitivities and specificities. The test, nonetheless, is more suitable for the evaluation of $H$. pylori infection eradication or in follow-up after a subsequent antimicrobial therapy [21]. The sensitivity of the test was assumed quite well on post-therapy, however; the test was not included in the study since the study was not aimed at post-therapy patient [21, 22].

Moreover, in low resource settings, the RUT (as an invasive test) and stool antigen (as a non-invasive test) may have an important role in clinical management of the infection. However, owing to, the low validity in predicting $H$. pylori infection, the stool antigen testing could be the crucial diagnostic test in follow up patient-like urea breath test. Ultimately, which in-turn decreases the number of patients requiring invasive tests, and cost as well [23]. Likewise, for a rapid and presumptive diagnosis, RUT could be another choice for the clinicians due to its comparable sensitivities to gold standard test, in detection of the pathogen-particularly low-income countries where molecular testing may not be assessable to everyone. 


\section{Conclusions}

In limelight, due to a protean clinical presentation and non-specific endoscopic findings; the dyspeptic syndrome is of pivotal concern for the clinicians. The accuracy order of the diagnostic tests may vary. Therefore, in reference to low-income countries, it is obligatory to resort any available positive test so that the supporting positive rudiments would be an auxiliary in augmenting the diagnostic precision.

\section{Limitation of the study}

The risk factor assessments, assessments of molecular methods in diagnosis, and follow-up treatment outcomes could have done; however, was not possible, owing to lack of resources and funding, in our setting.

\section{Abbreviations}

ELISA: enzyme-linked immune sorbent assay; FD: functional dyspepsia; HpSAg: Helicobacter pylori stool antigen; H. pylori: Helicobacter pylori; PCR: polymerase chain reaction; RUT: Rapid Urease Test.

\section{Authors' contributions}

GC, PK conceived the study, design the manuscript, review of the literature. GM and PP reviewed the manuscript and gave the concept of the research paper and critically reviewed the manuscript. All authors read and approved the final manuscript.

\section{Author details}

${ }^{1}$ Trichandra Multiple Campus, Tribhuvan University, Ghantaghar, Kathmandu, Nepal. ${ }^{2}$ Nepal National Hospital, Kathmandu, Nepal.

\section{Acknowledgements}

We would like to thanks Asst. Prof. Shyam Kumar Mishra (Institute of Medicine, TUTH) for his tremendous technical support.

\section{Competing interests}

The authors declare that they have no competing interests.

\section{Availability of data and materials}

All data generated or analyzed during this study are included in this published article.

\section{Consent for publication}

Not applicable.

\section{Ethics approval and consent to participate}

This study was approved by the local ethical committee of the Nepal National Hospital. Written informed consent was obtained from every patient for granting participation in an interview and to extract pertinent socio-demographic and clinical data from their respective clinical files, respecting confidentiality.

\section{Funding}

No monetary benefit was received for this study.

\section{Publisher's Note}

Springer Nature remains neutral with regard to jurisdictional claims in published maps and institutional affiliations.

Received: 3 September 2018 Accepted: 30 October 2018

Published online: 03 November 2018
References

1. Helicobacter pylori. IARC monographs on the evaluation carcinogenic risks to humans. 1994;61:177-240. https://monographs.iarc.fr/wp-content/ uploads/2018/06/mono100B-15.pdf.

2. McColl KEL. Clinical practice Helicobacter pylori infection. N Engl J Med. 2010;362(17):1597-604

3. Malfertheiner P, Megraud F, O'Morain CA, et al. Management of Helicobacter pylori infection - the Maastricht IV/Florence consensus report. Gut. 2012;61:646-64.

4. Moayyedi P, Forman D, Braunholtz D, et al. The proportion of upper gastrointestinal symptoms in the community associated with Helicobacter pylori, lifestyle factors, and nonsteroidal anti-inflammatory drugs. Am J Gastroenterol. 2000:95:1448-55.

5. Bazzoli F, De Luca L, Pozzato P, Zagari RM, Fossi S, Ricciardiello L, et al. Helicobacter pylori and functional dyspepsia : review of previous studies and commentary on new data. Gut. 2002;50:33-5.

6. Zullo A, Hassan C, De Francesco V, Repici A, Manta R, Tomao S, et al. Helicobacter pylori and functional dyspepsia: an unsolved issue? World J Gastroenterol. 2014;20(27):8957-63.

7. Mahadeva SGK. Epidemiology of functional dyspepsia: a global perspective. World J Gastroenterol. 2006;12:2661-6.

8. Mégraud F, Lehours P. Helicobacter pylori detection and antimicrobial susceptibility testing. Clin Microbiol Rev. 2007;20(2):280-322.

9. Isenberg HD. Clinical microbiology procedures handbook. 2nd ed. Washington, DC: ASM Press; 2004.

10. Khalifehgholi M, Shamsipour F, Ajhdarkosh H, Daryani NE, Reza Pourmand M, Hosseini M, et al. Comparison of five diagnostic methods for Helicobacter pylori. Iran J Microbiol. 2013;5(4):396-401.

11. Salih BA. Helicobacter pylori infection in developing countries: the burden for how long? Saudi J Gastroenterol. 2009;15(3):201-7.

12. Pk B. Epidemiological features of Helicobacter pylori infection in developing countries. Clin Infect Dis. 1997;25(5):973-8.

13. Archampong TNA, Asmah RH, Wiredu EK, Gyasi RK, Nkrumah KN, Rajakumar K. Epidemiology of Helicobacter pylori infection in dyspeptic Ghanaian patients. Pan Afr Med J. 2015;20:1-9.

14. Rosenstock SJ, Andersen LP, Rosenstock CV, Bonnevie O, Jørgensen T. Socioeconomic factors in Helicobacter pylori infection among Danish adults. Am J Public Health. 1996:86(11):1539-44.

15. Chen HL, Chen MJ, Shih SC, Wang HY, Lin IT, Bair MJ. Socioeconomic status, personal habits, and prevalence of Helicobacter pylori infection in the inhabitants of Lanyu. J Formos Med Assoc. 2014;113(5):278-83. https //doi.org/10.1016/j.jfma.2013.11.013.

16. Ansari S, Gautam R, Nepal HP, Subedi SN, Shrestha S, Mandal F, et al. Helicobacter pylori colonization in Nepal; Assessment of prevalence and potential risk factors in a hospital-based patient cohort microbiology. BMC Res Notes. 2016:9(1):4-9.

17. Miftahussurur M, Sharma RP, Shrestha PK, Suzuki R, Uchida T, Yamaoka Y. Molecular epidemiology of Helicobacter pylori infection in Nepal: specific ancestor. PLoS One 2015;10(7):e0134216. https://doi.org/10.1371/journ al.pone.0134216.

18. El-Omar EM, Penman ID, Ardill JE, Chittajallu RS, Howie C, McColl KE. H. pylori infection and abnormalities of acid secretion in patient with chronic duodenal ulcer disease. Gastroenterology. 1995;109:681-91.

19. Srinivas Y, Prasad PK, Sai ND. Prevalence and impact of Helicobacter pylori in dyspepsia. Int Surg J. 2016:3(1):305-9.

20. $\operatorname{Tr}$ O. Upper gastrointestinal endoscopic findings in adolescents at Lacor hospital, Uganda. Afr Health Sci. 2006;6(1):39-42.

21. Opekun AR, Gotschall AB, Abdalla N, Agent C, Torres E, Sutton FM, Graham DYTK. Improved infrared spectrophotometer for point-of-care patient 13C-urea breath testing in the primary care setting. Clin Biochem. 2005;38:731-4

22. Patel SK, Pratap CB, Jain AK, Gulati AK, Nath G. Diagnosis of Helicobacter pylori: what should be the gold standard? World J Gastroenterol. 2014:20(36):12847-59.

23. Segamwenge IL, Kagimu M, Ocama P, Opio K. The utility of the Helicobacter pylori stool antigen test in managing dyspepsia : an experience from a low resource setting. Afr Health Sci. 2014;14(4):4-7. 